\title{
Infection Prevention and Control in Combating Antimicrobial Resistance in Sudan
}

\section{Ghada Omer Shouna ${ }^{1 *}$, Maye Abu Omar ${ }^{2}$, Samira Hamid Abdelrahman ${ }^{3}$ and Gamal Khalafalla Mohammed ${ }^{4}$}

${ }^{1}$ University of Gezira, Sudan

${ }^{2}$ Nuffield Centre for International Health and Development, University of Leeds, UK

${ }^{3}$ Department of Family and Community Medicine, University of Gezira, Sudan

${ }^{4}$ Pamela Steele Associates Ltd, Ethiopia

\section{Research Article \\ Volume 4 Issue 2}

Received Date: September 11, 2020

Published Date: October 15, 2020

DOI: $10.23880 /$ phoa-16000160

*Corresponding author: Ghada Omer Shouna, University of Gezira, Madani, Sudan, Email: ghadashouna@hotmail.com

\section{Abstract}

Antimicrobial resistance (AMR) has been a priority area identified by the Government of Sudan and development partners. This paper is part of a study in Sudan using the available documents covering relevant fields pertaining to AMR and interviews with key informants from World Health Organization office Sudan, Federal Ministry of Health, Ministry of Environment and Ministry of General Education. Documents were obtained from relevant websites for policies, strategies, guidelines, reports, and plans as well relevant international and government agencies in Khartoum. The study was guided by the World Health Organization's five strategic objectives of Global Action Plan on Antimicrobial Resistance. This paper focuses on the third objective on reducing the incidence of infection through effective sanitation, hygiene and infection prevention measures. The findings reveal, among others, weak collaboration among the relevant sectors to play their role in infection control and poor adherence to existing guidelines especially at health facilities in addition to scarcity of resources in terms of soap, water and other means to safeguard against infection. The study revealed that training workshops, regulatory measures, education and raising the awareness of the public play a complementary role in reducing infections. In conclusion, there is a need for strong collaboration between different ministries to deal with the problem of AMR under the concept of 'one health'. Similarly, establishing regulatory measures enhancing compliance with the existing guidelines and the development of a roadmap to establish a national AMR surveillance system in Sudan and setting up laboratory-based nationwide surveillance of antimicrobial resistance is essential in order to establish a robust system to tackle the problem of AMR in the country.

Keywords: Antimicrobial Resistance; Health Facilities; Infection Control; Sudan; Training

Abbreviations: AMR: Antimicrobial Resistance; AST: Antimicrobial Susceptibility Testing; EFCD: Environmental \& Food Control Department; FAO-OIE-WHO: Food and Agricultural Organization-World Organization for Animal Health-World Health Organization; FMoH: Federal Ministry of Health; GAP: Global Action Plan; ICC: Infection Control
Committee; JEE: Joint External Evaluation; IHR: International Health Regulations; IPC: Infection Prevention and Control; KSMoH: Khartoum State Ministry of Health; MOEN: Ministry Of Environment and Natural Resources; MOGE: Ministry Of General Education; WHA: World Health Assembly; WHO: World Health Organization. 


\section{Introduction}

Antibiotics are valuable therapeutic agents that brought many infectious diseases under control $[1,2]$. Today, they are indispensable in all health systems. Achievements in modern medicine, such as major surgery, organ transplantation, cancer chemotherapy and even some viral infections would not be possible without access to effective antibiotics [3]. Unfortunately, the imprudent use of these medicines is bringing their miraculous effect to an end and the rapid development of resistance is threatening heath security globally. Antimicrobial resistance (AMR) is increasingly recognised as a key public health concern for both developed and developing countries due to its potentially alarming socioeconomic impact on health [4].

The control of infectious diseases is seriously threatened by the steady increase in the number of microorganisms that are resistant to antimicrobial agents. Resistant infections adversely affect mortality, treatment costs, disease spread, duration of illness, and increase cost to health systems [5,6]. Although antimicrobial resistance is a natural phenomenon, it is being propagated by misuse of antimicrobial medicines, inadequate or inexistent programmes for infection prevention and control (IPC), poor-quality medicines, weak laboratory capacity, inadequate surveillance and insufficient regulation of the use of antimicrobial medicines $[7,8]$. Surveillance of AMR is now one of the components of International Health Regulations (IHR). In 2012, the World Health Assembly (WHA65.23) urged State Parties to take the necessary steps to prepare and carry out appropriate national implementation plans in order to ensure the required strengthening [9], development and maintenance of the core public health capacities as provided for in the IHR [10]. In 2016, a multi-sectoral International Evaluation Team conducted a joint assessment of IHR core capacities of Sudan using the World Health Organization's (WHO) International Health Regulation (IHR) Joint External Evaluation (JEE) tool [11]. The assessment concluded that capacities for combating AMR scored low. The indicators included antimicrobial resistance detection, surveillance of infections caused by resistant pathogens, healthcare associated infection prevention and control programmes, and antimicrobial stewardship activities [1,12].

On the other hand, a significant information gap was identified in several areas during a Joint Food and Agricultural Organization-World Organization for Animal Health-World Health Organization (FAO-OIE-WHO) consultation on AMR in Sudan towards developing a national action plan on AMR [13]. It was deemed necessary to gather information for use in developing a national action plan on AMR for Sudan, which led to undertaking a study that was guided by the
WHO's five strategic objectives of Global Action Plan (GAP) on Antimicrobial Resistance (Figure 1) [14].

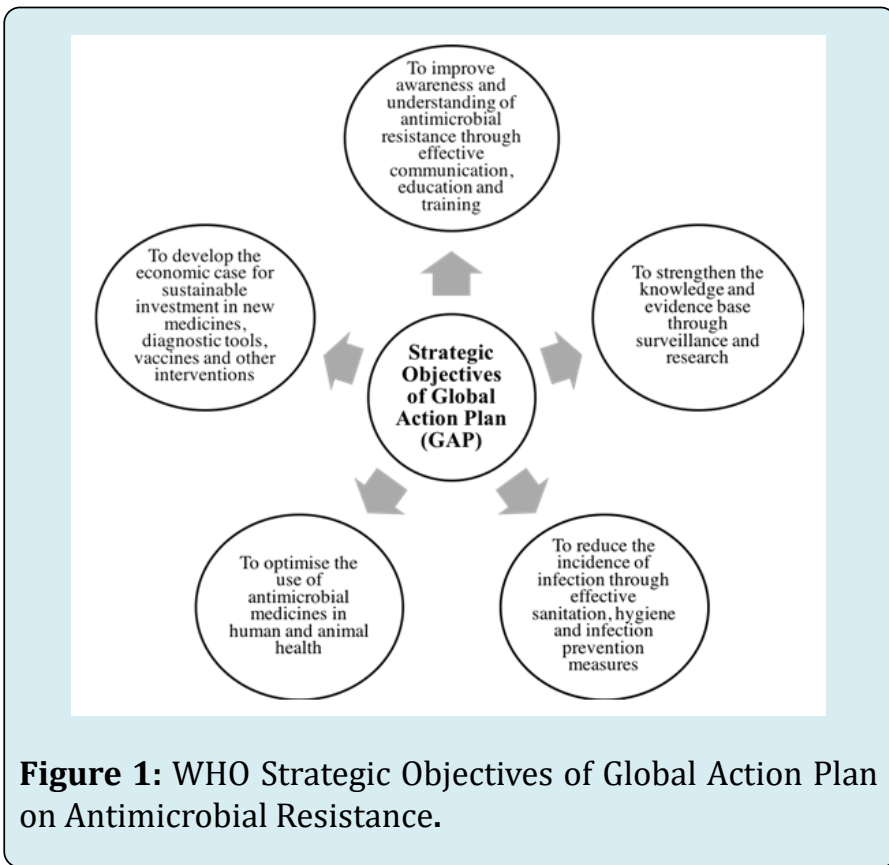

This paper focuses on the third objective, i.e. reduction of the incidence of infection through effective sanitation, hygiene and infection prevention measures.

\section{Methods}

The study comprised a series of methods, which involved meetings and interviews with key informants from the World Health Organization (WHO) Country Office and other governmental institutions such as health, environment and education among others in Khartoum that play a role in combating AMR, and reviewing the relevant documents available pertaining to the subject of AMR in Sudan. Interviews were conducted with key informants using a questionnaire guide to obtain information about surveillance system to provide data on antimicrobial susceptibility, hygiene, infection prevention and control measures in health facilities and wider community in regard to reducing the incidence of infection through effective sanitation, hygiene and infection prevention. During the interviews, the study explored measures and programmes that were in place to ensure hygiene and infection control in health facilities, and how policies and programmes were monitored and evaluated. Data from key informants was used to supplement and complement information obtained from the documents. The documents reviewed included strategic plans, national and sectoral policy documents and mission reports available at WHO office in Sudan, Federal Ministry of Health (FMoH), 
Khartoum State Ministry of Health (KSMoH), and Ministry of General Education (MOGE), which were either obtained physically or from various websites [14].

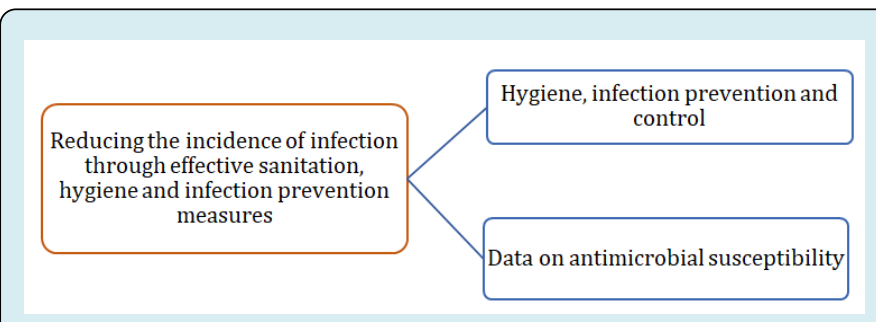

Figure 2: Components of objective 3 of GAP on Antimicrobial Resistance.

As illustrated in Figure 2, in order to address objective 3 of GAP, the study considered the availability of a surveillance system to provide data on antimicrobial susceptibility and measures that are in place to promote hygiene and infection control. In this regard, information from the document review and the interviews was collated to identify gaps that need to be addressed in line with the strategic objectives of the WHO GAP on Antimicrobial Resistance.

\section{Findings}

\section{Hygiene, infection prevention and control}

Better hygiene and infection prevention measures are essential to limit the development and spread of antimicrobial resistant infections and multidrug-resistant bacteria as well as reducing the need to use antimicrobial agents. Standards of practice regarding infection prevention and control at heath facilities in Sudan were reported to be poor despite the existence of policies, guidelines and training activities. At FMOH level, an Infection Control Unit, which is the executive arm of the National Standing Committee on Infection Control (ICC), has been established. This Unit has contributed in drafting a National Infection and Control (IPC) plan and manual. The Unit has also organised training of trainers and a number of workshops on infection prevention and control for health staff in collaboration with the 'Arab Institute for Continuous Professional Development' to disseminate the IPC strategies and messages.

The study found that a number of health facilities in Khartoum State have IPC programmes in place. However, at country level, implementation of IPC in health care facilities is reported by key informants as a concern due to lack of adherence of health care workers to infection control measures. This is a result of insufficient training and funding, lack of monitoring and supervision mechanisms as well as lack of resources that ensure availability of soap and reliable water supply and other means to safeguard against infection.

On the other hand, the Ministry of Environment, Natural Resource and Physical Development (MOEN) and other departments such as the Environmental \& Food Control Department (EFCD) of the Federal Ministry of Health (FMOH) plays an important role in this matter. The EFCD, FMoH has developed guidelines for disposal of Primary Health Care (PHC) medical waste. The EFCD Has also developed guidelines on drinking water safety, which address the issue of microbial contamination as the first safety standard. A key informant from FMoH reported " $E F C D$, FMOH has guidelines on drinking water safety and guidelines on Health requirements for food stores, which are extremely important to control food safety hazards that are associated with microbial contamination". However, guidelines may not fulfil their purpose because of limited dissemination, lack of proper implementation and monitoring by the EFCD units at State level.

Having a clean healthy environment will have a direct impact on the prevention of infection. A number of gaps have been identified from the available documents in this area. These include:

- Absence of mechanisms to monitor microorganisms and antibiotic residues in soil and water.

- Lack of regulations for detection and prevention of antibiotic resistance development at the Environmental \& Food Control Department (EFCD).

- Lack of information on the extent of enforcement and compliance to the available guidelines.

The MOEN, the body responsible for wastewater treatment, has a vital role in ensuring a clean environment through its municipalities in Sudan. The study, found that there was a serious problem of hygiene, cleanliness and waste disposal throughout Khartoum State. The study found that the MOEN had no activities pertaining to minimising the spread of antimicrobials through the environment.

\section{Data on Antimicrobial Susceptibility}

Official data on antimicrobial susceptibility of microorganisms is not widely available in the country. As illustrated in Table 1, a number of studies have been conducted for different purposes to study resistance patterns. Although those studies are quite limited, they nevertheless have their importance and relevance in regard to the problem of antimicrobial susceptibility. However, the key stakeholders reported that the findings of the studies have not been disseminated to the relevant bodies to effect in decision making. 


\begin{tabular}{|c|c|}
\hline Studies and type & Summary of key findings \\
\hline $\begin{array}{l}\text { Kheder SI, Cephalosporins usage and resistance trend in a } \\
\text { Sudanese hospital surgical ward [15] }\end{array}$ & $\begin{array}{l}90-100 \% \text { of Enterobacteriacea isolates in Ibn-Sina Hospital } \\
\text { during } 2008-2010 \text { were resistant to } 3^{\text {rd }} \text { generation } \\
\text { cephalosporins. }\end{array}$ \\
\hline $\begin{array}{l}\text { Ali MA, et al. Studies on the prevalence and } \\
\text { characterization of antibiotic resistance among gram- } \\
\text { negative bacilli Khartoum, Sudan: PhD. Thesis [16] }\end{array}$ & $\begin{array}{l}\text { Resistance of E. Coli and Klebsiella spp to ceftriaxone and } \\
\text { ceftazidime in Khartoum Teaching Hospital and Soba University } \\
\text { Hospital ranged from 56.5-79\% respectively. }\end{array}$ \\
\hline $\begin{array}{l}\text { Basheer NA, et al. Antibiotic usage and prevalence of } \\
\text { antibiotic resistance in Soba Hospital Audit Report [17] }\end{array}$ & $\begin{array}{l}70 \% \text { of } E \text {. Coli and } 80 \% \text { of } K \text { pneumoniae respectively were ESBL } \\
\text { producers. } 51 \% \text { of Staph Aureus were MRSA. }\end{array}$ \\
\hline $\begin{array}{l}\text { Yousif MA, Prevalence of ESBLs and AmpC beta lactamases } \\
\text { among Gram-negaive clinical isolates in Soba Teaching } \\
\text { Hospital. Khartoum, Sudan: M.D. Thesis [18] }\end{array}$ & $\begin{array}{l}61.9 \% \text { of } E \text {. Coli and } 83.8 \% \text { of } K \text { pneumoniae in Khartoum } \\
\text { hospitals were ESBL producers. }\end{array}$ \\
\hline \multirow[t]{5}{*}{ Khartoum State. Microbiology Records 2013 [19] } & $\begin{array}{l}\text { - MRSA ranged between } 29 \% \text { and } 80 \% \text { in Khartoum Teaching } \\
\text { Hospital and Soba University Hospital. }\end{array}$ \\
\hline & $\begin{array}{l}\text { - Resistance to Enterobacteriaceae } 3^{\text {rd }} \text { generation cephalosporins } \\
\text { ranged from } 61-88 \% \text { in Khartoum Teaching Hospital and Soba } \\
\text { University Hospital. }\end{array}$ \\
\hline & $\begin{array}{l}\text { - 3-5\% of Pseudomonas spp and non-fermenters were resistant } \\
\text { to carbapems. }\end{array}$ \\
\hline & $\begin{array}{l}\text { - Prevalence of vancomycin-resistant } S . \text { aureus was found to be } \\
2 \% \text { in Soba University Hospital. }\end{array}$ \\
\hline & $\begin{array}{l}\text { - Vancomycin-resistant enterococcus ranged from } 21 \% \text { in Soba } \\
\text { University Hospital to } 33 \% \text { at the National Health Laboratory. }\end{array}$ \\
\hline
\end{tabular}

Table 1: Summary of studies showing antimicrobial susceptibility of microorganisms.

In some health facilities, such as Soba teaching hospital, culture and sensitivity testing were conducted to monitor the resistance pattern which is encouraging. On the other hand, about 33\% of public hospitals at Khartoum State reported conducting culture and sensitivity testing, yet no data was available on the nature of the analysis. Similarly, no data was available from private hospitals and private medical laboratories.

Overall, there is lack of reliable data on antimicrobial susceptibility of microorganisms due to lack of a monitoring and reporting system. Likewise, the available data on culture and sensitivity testing conducted by a few hospitals is rarely used to monitor AMR pattern in those facilities.

\section{Discussion and Conclusion}

The most serious and difficult-to-treat antibioticresistant infections occur in health care facilities, not only because that is where patients with serious infections are treated but also because of the intensive use of antibiotics [20]. Although the development of resistance in such situations may be a natural consequence of necessary antimicrobial use, the study has shown that measures to prevent and control infection that may contribute to the spread of microorganisms resistant to antimicrobial medicines are inadequate in Sudan.

Poor or ineffective measures to promote hygiene and infection prevention in health facilities and the natural environment, in general, are regarded as pathways by which transmission of antibiotic residues to humans might occur and that could contribute to the development of AMR. Antibiotic residues in the environment may originate from wastewater, the soil, manure and other food waste. The accumulation of these compounds in the environment, and the impact of such accumulation on the emergence of antibiotic resistance should not be underrated [21]. Similarly, resistant organisms disseminate from humans to animals, and vice versa, often through various environmental pathways, including foodstuffs, animal wastes, and water sources [22]. The study has shown that there is no system or programme to detect antibiotic residues or to block the drivers and pathways of AMR in the environment. Hygiene, cleanliness and waste disposal systems were found to be poor. Therefore there is a need for appropriate intervention strategies for controlling AMR in the environment to limit the emission of resistant strains of bacteria, and strategies to mitigate the potential transmission of antimicrobial resistance to humans [23]. A well-functioning and efficient waste disposal and wastewater 


\section{Public Health Open Access}

treatment system is mandatory for effective preventive measures and environmental protection from AMR. Water quality measures should not only focus on the presence of microorganisms but should also address the antibiotic concentrations in sewages and treatment plants [24].

Sound hygiene and infection prevention measures can limit the spread of resistant microorganisms and reduce antimicrobial misuse and overuse. Such measures will include hand hygiene, appropriate aseptic techniques, maintenance of clean, hygienic medical equipment along with thoughtful and thorough surveillance, monitoring and evaluation measures [25]. Hand hygiene is now regarded as one of the most important elements of infection control activities. If properly implemented, hand hygiene alone can significantly reduce the risk of cross-transmission of infection in healthcare facilities [26]. Therefore, hygiene and hand washing should be enforced at health facilities. Moreover, it should be a core component of school education to influence behaviour at early age [27].

Data on antimicrobial susceptibility is important for both medical decision-making and policy-making. Unfortunately, the study has shown that, data availability for decision and policy making is limited and remains a challenge in Sudan despite the unprecedented advances in technology over the past several decades. This is most apparent when it comes to empirical and targeted treatment with antibiotics. Years of antibiotic misuse and overuse have led to global resistance and the rise of new bacterial resistance threats that defy treatment with conventional therapies. Antimicrobial susceptibility testing (AST) confirms susceptibility, detects resistance and guides the rational selection of patient therapy. Without solid and reliable data on AST of micro-organisms no rational decisions or actions could be effected to tackle the problem of AMR in the country [28]. Information on local patterns of antimicrobial susceptibility can be collected using AST, so that policies guiding the empiric choice of therapy can be based on data on local resistance trends (also known as the local or institutional antibiogram). AST can also help to identify isolates with defined resistance mechanisms of major interest to infection prevention and control.

A system on data collection, analysis and dissemination is urgently required. The validity and reliability of the data depends on the quality of the medical laboratories, equipment and material used, staff skills and testing techniques. For reliable results, it is suggested to establish an accreditation system for medical laboratories in Sudan. This will ensure the application of standardised techniques and the production of reliable data that can be used for making the necessary medical and policy decisions [29]. Microbiology labs are therefore essential components in stewardship programs to ensure quality specimen collection, appropriate testing, and implementation of rapid diagnostics, AST and data analysis. Recognizing the value of this tool and working collaboratively with laboratories-particularly in high-risk patients is a cornerstone of appropriate and targeted therapy and generation of reliable susceptibility data [30].

In conclusion, the importance of collaboration between the ministries of education, environment and health under the concept of 'One Health' need to be promoted. MOEN needs to establish regulatory measures to control the emission of resistant bacteria strain and antimicrobial residues in the environment. Hand washing and other measures for infection control, for example should be a core component of school education to influence children in forming a behaviour that then becomes a habit and eventually a pattern. Similarly, enhancing compliance with the existing guidelines and the development of a roadmap to establish a national AMR surveillance system in Sudan and setting up laboratorybased nationwide surveillance of antimicrobial resistance is essential in order to establish a robust system to tackle the problem of AMR in the country.

\section{References}

1. Shouna GO, Omar MA, Abdelrahman SH (2020) Advancing awareness on antimicrobial resistance: A situation analysis in Sudan. European Journal of Biomedical and Pharmaceutical Sciences 7(8): 112-120.

2. Fair RJ, Tor Y (2014) Antibiotics and bacterial resistance in the 21st century. Perspect Medicin Chem 6: 25-64.

3. Aminov RI (2010) A brief history of the antibiotic era: lessons learned and challenges for the future. Front Microbiol 1: 134.

4. Shrestha P, Cooper BS, Coast J, Oppong R, Do Thi Thuy $\mathrm{N}$, et al. (2018) Enumerating the economic cost of antimicrobial resistance per antibiotic consumed to inform the evaluation of interventions affecting their use. Antimicrob Resist Infect Control 7: 98.

5. Hecke VO, Wang K, Lee JJ, Roberts NW, Butler CC (2017) Implications of Antibiotic Resistance for Patients' Recovery From Common Infections in the Community: A Systematic Review and Meta-analysis. Clin Infect Dis 65(3): 371-382.

6. Shouna GO, Omar MA, Abdelrahman SH (2020) Strengthening knowledge and evidence to combat antimcrobial resistance in Sudan. European Journal of Biomedical and Pharmaceutical Sciences 7(8): 145-150.

7. Saust LT, Monrad RN, Hansen MP, Arpi M, Bjerrum L (2016) Quality assessment of diagnosis and antibiotic 
treatment of infectious diseases in primary care: a systematic review of quality indicators. Scand J Prim Health Care 34(3): 258-266.

8. Costelloe C, Metcalfe C, Lovering A, Mant D, Hay AD (2010) Effect of antibiotic prescribing in primary care on antimicrobial resistance in individual patients: systematic review and meta-analysis. Bmj 340: c2096.

9. WHO (2012) Report of the Executive Board on its 129th, 130th and special sessions. Sixty-fifth World Health Assemby, pp: 1-6.

10. WHO (2008) Implementation of the International Health Regulations (2005). Sixty-fifth World Health Assembly, World Health Organization, pp: 1-2.

11. WHO (2018) Joint External Evaluation tool (JEE tool)second edition IHR (2005) Monitoring and Evaluation framework. World Health Organization.

12. WHO (2016) Joint External Evaluation (JEE) of Sudan Mission Report. World Health organization.

13. WHO (2017) GoS, WHO, FAO, and OIE fight antimicrobial resistance with new national plan. World Health Organization.

14. WHO (2015) Global action plan on antimicrobial resistance. World Health Organization.

15. Kheder S (2011) Cephalosporins usage and resistance trend in a Sudanese hospital surgical ward. Journal of Pharmaceutical and Biomedical Sciences 11(3): 1-7.

16. Ali MA (2013) Studies on the prevalence and characterization of antibiotic resistance among gramnegative bacilli Khartoum. PhD. Thesis, University of Khartoum, Sudan.

17. Basheer NA, Elhag KM (2013) Antibiotic usage and prevalence of antibiotic resistance in Soba Hospital Audit. Study Report. Khartoum, Sudan.

18. Yousif MMA (2013) Prevalence of ESBLs and AmpC beta lactamases among Gram-negaive clinical isolates in Soba Teaching Hospital. MD Thesis, Sudan Medical Specialization Board, Khartoum, Sudan.

19. (2013) Microbiology Records, Khartoum State, Khartoum.

20. Irek EO, Amupitan AA, Obadare TO, Aboderin AO (2018) A systematic review of healthcare-associated infections in Africa: An antimicrobial resistance perspective. Afr J Lab Med 7(2): 796.

21. Roca I, Akova M, Baquero F, Carlet J, Cavaleri M, et al. (2015) The global threat of antimicrobial resistance: science for intervention. New Microbes and New Infections 6: 22-29.

22. Finley RL, Collignon P, Larsson DG, McEwen SA, Li XZ, et al. (2013) The scourge of antibiotic resistance: the important role of the environment. Clin Infect Dis 57(5): 704-710.

23. Singer AC, Shaw H, Rhodes V, Hart A (2016) Review of Antimicrobial Resistance in the Environment and Its Relevance to Environmental Regulators. Front Microbiol 7: 17-28.

24. Barancheshme F, Munir M (2018) Strategies to Combat Antibiotic Resistance in the Wastewater Treatment Plants. Front Microbiol 8: 2603.

25. WHO (2018) Improving infection prevention and control at the health facility: interim practical manual supporting implementation of the WHO guidelines on core components of infection prevention and control programmes. World Health Organization.

26. Mathur P (2011) Hand hygiene: Back to the basics of infection control. Indian J Med Res 134(5): 611-620.

27. Snow M, White GL, Kim HS (2008) Inexpensive and timeefficient hand hygiene interventions increase elementary school children's hand hygiene rates. J Sch Health 78(4): 230-234.

28. Seale AC, Gordon NC, Islam J, Peacock SJ, Scott JAG (2017) AMR Surveillance in low and middle-income settings-A roadmap for participation in the Global Antimicrobial Surveillance System (GLASS). Wellcome Open Res 2: 92.

29. Perilla MJ (2003) Manual for the laboratory identification and antimicrobial susceptibility testing of bacterial pathogens of public health importance in the developing world; Haemophilus influenzae, Neisseria meningitidis, Streptococcus pneumoniae, Neisseria gonorrhoeae, Salmonella serotype Typhi, Shigella, and Vibrio cholerae. World Health Organization.

30. Belkum VA, Bachmann TT, Ludke G, Lisby JG, Kahlmeter G, etal. (2019) Developmental roadmap for antimicrobial susceptibility testing systems. Nat Rev Microbiol 17(1): 51-62. 